\title{
Performance Evaluation of Fire Alarm System Based on FPNN*
}

\author{
Wei Gou ${ }^{1, a}$, $\mathrm{Na} \mathrm{Qu} \mathrm{u}^{1, \mathrm{~b}}{ }^{*}$ and Zhenliang Lan ${ }^{1, \mathrm{c}}$ \\ 1 School of Safety Engineering, Shenyang Aerospace University, Shenyang 110136, China. \\ a 1960100041@qq.com, b11502332@qq.com, c1140378766@qq.com
}

Keywords: performance evaluation; fire alarm system; fuzzy mathematics; probabilistic neural networks;

\begin{abstract}
The type of fire alarm system and grades of standards exist fuzziness. fuzzy mathematics relative membership grade theory and probabilistic neural networks are combined to build a fuzzy probabilistic neural network (FPNN) evaluation model of fire alarm system. The method of constructing model is proposed based on indicators relative membership degree matrix interpolation. By comparing with other methods, the model is verified to be effective and the result is objective and reliable.
\end{abstract}

\section{Introduction}

The performance of the automatic fire alarm system is very important because it has an effect on the early warning ability of the fire, the ability of the alarm and the ability to extinguish the fire. In the paper, the new Chinese national standard GB50116-2013 "automatic fire alarm system design specifications" and national guidance documents GB / Z24978-2010 "automatic fire alarm system performance evaluation" is analyzed. Product performance includes reliability, environmental adaptability, compatibility, maintainability, scalability, ease of use, etc. At present, the research on the performance of the automatic fire alarm system is mainly aimed at the reliability of the fire alarm system and other properties are less considered.

In 2009, Zhao Hairong analyzed the method of system failure risk based on fault tree and summarized the factors affecting the reliability of the system [1]. Wang Qingxi analyzed the causes of urban tunnel fire and the established model of BP neural network evaluation [2]. Zuo Lai established the evaluation indexes of fire alarm system by using fuzzy algorithm and genetic algorithm [3]. In 2012, Xing Zhixiang calculated the reliability of fire alarm system by numerical calculation method [4].

\section{FPNN evaluated using an automatic fire alarm performance}

\section{A Selection and evaluation of grading standards}

When evaluating the system or other components, the six categories as the first level evaluation index are selected, including reliability, environmental adaptability, compatibility, maintainability, scalability and ease of use.

\section{B Quantitative assessment indicators}

The performance evaluation index system of fire alarm system can only be used to describe the performance of the system, and cannot be described by the exact numerical value. These evaluation indexes have some fuzziness. This evaluation index performance can be represented by a number of

* College students training program of innovation and entrepreneurship in Shenyang Aerospace University DS504101 
figures by expert scoring. The expert scoring method is mainly based on the practical experience or by the calculation of the theorem formula[5].

Table1. Evaluation of fire alarm system product classification standard

\begin{tabular}{ccccccc}
\hline Standard & Reliability & $\begin{array}{c}\text { Environmental } \\
\text { Adaptability }\end{array}$ & Serviceability & Scalability & Accessibility Compatibility \\
\hline I & $0.9 \sim 1$ & $0.9 \sim 1.1$ & $8 \sim 10$ & $9 \sim 10$ & $7 \sim 10$ & $9 \sim 10$ \\
\hline II & $0.75 \sim 0.9$ & $0.8 \sim 0.9$ or 1.1 1.2 & $6 \sim 8$ & $6 \sim 9$ & $6 \sim 7$ & $8 \sim 9$ \\
\hline III & $0.6 \sim 0.9$ & $0.7 \sim 0.8$ or 1.2 1.3 & $5 \sim 6$ & $4 \sim 6$ & $3 \sim 5$ & $6 \sim 8$ \\
\hline IV & $0.4 \sim 0.6$ & $0.5 \sim 0.7$ or 1.3 1.5 & $3 \sim 5$ & $3 \sim 4$ & $1 \sim 2$ & $4 \sim 6$ \\
\hline V & $0 \sim 0.4$ & $0 \sim 0.5$ or $\geqslant 1.5$ & $0 \sim 3$ & $0 \sim 3$ & $0 \sim 1$ & $0 \sim 4$ \\
\hline
\end{tabular}

\section{Probabilistic neural network model}

Probabilistic neural network was proposed by Specht. It is closely linked with statistical signal processing and similar in structure to back-propagation network. The main difference lies in the use of statistical methods to deduce the incentive function instead of the traditional neural network Sigmoid type activation function. The theory of probabilistic neural network is based on a Bayesian decision theory [6][7].

\section{Realization of probabilistic neural network by MATLAB}

The PNN neural network toolbox of MATLAB contains many functions for PNN analysis and design.

(1)newpnn(P,T,SPREAD): It can quickly create a probabilistic neural network.

(2)radbas( ): The output of the neuron can be obtained by its network input.

(3) dist (W, P): The function of the input is weighted by the input. In the PNN layer, the input feature vector is computed and the matching relationship between the training set and the pattern of the training set, and the transfer function and the Gauss type activation function are obtained.

(4) train (net, P, T): Neural network training function.

(5) $\operatorname{sim}($ net, $P$ ): Neural network simulation functions.

\section{Engineering examples}

According to the evaluation and classification of fire automatic alarm system, 30 learning samples and 15 test samples are selected. The reliability, environmental adaptability, maintainability, scalability, ease of use and compatibility are selected for the input vector. The number of neurons in the input layer is six. The output is evaluation class that has five level.

Newpnn (P, T) function in Matlab neural network toolbox can be used to establish the PNN model and SIM (net, P) function can be used to simulate, after the original data is dealed with fuzzy algorithm. After multiple parameter optimization operation, the smoothing parameter of the best effect of the Gauss function is 0.5 to 0.4 .

The program of FPNN training and simulating is given.

load('s4. mat');

Train $=\mathrm{s} 4(1: 30,:)$;

Test $=$ s4 ( $31:$ end, : );

p_train=Train $(:, 1: 6)^{\prime}$;

t_train=Train $(:, 7) '$;

p_test=Test $(:, 1: 6)^{\prime}$ ';

t_test=Test $(:, 7) '$;

t_train=ind2vec(t_train);

t_train_temp=Train $(:, 7)^{\prime}$; ;

Spread $=0.45$;

net=newpnn (p_train,t_train, spread ) ; 


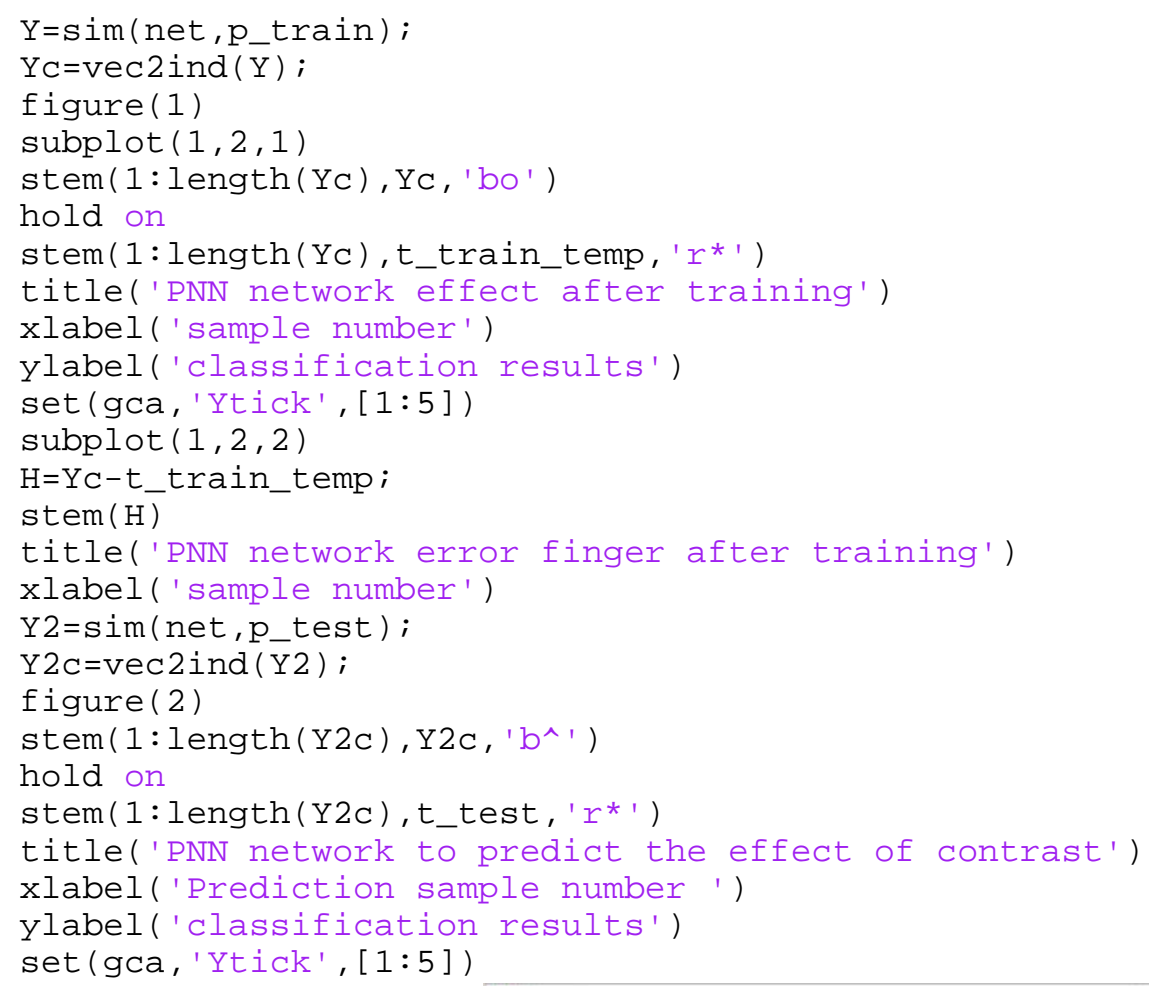

Fig.1 PNN network effects and error plots

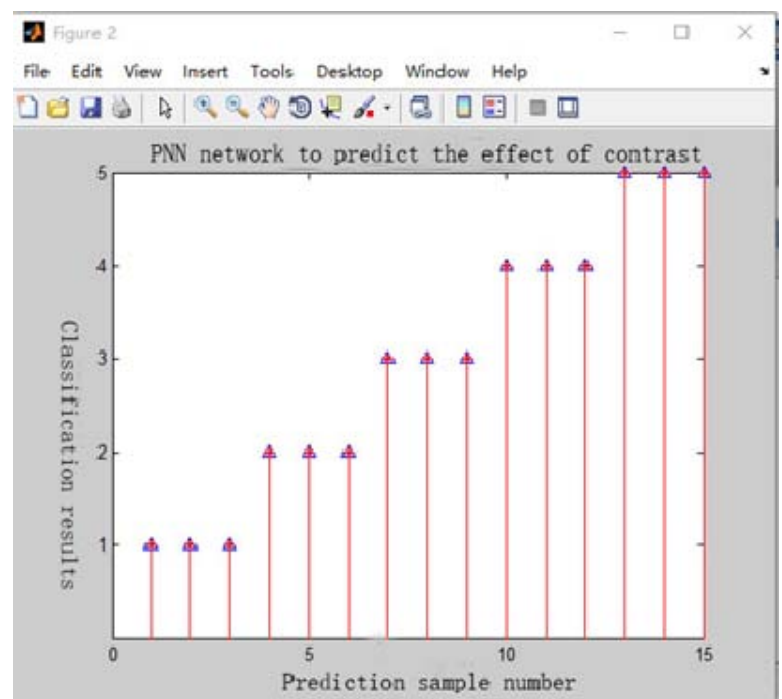

Fig. 2 the effect of contrast 


\section{Summary}

The performance of fire alarm system is evaluated by fuzzy probabilistic neural network (FPNN). The influence of various factors on the performance can be considered by the relative membership degree of fuzzy mathematics. The existence shortcomings of the maximum membership degree that only considered the extreme value and lost the intermediate information can be overcome. Probabilistic neural network is based on the Bayesian minimum risk criteria and the evaluation criteria can be used to evaluate the performance of the fire alarm system. The network training need not a large number of samples and the artificial regulation parameters are less. In the training, only the smoothing factor of the Gauss function is estimated. The training speed is fast and has a strong ability of fault tolerance and adaptive capacity. The classification ability is strong and the evaluation results are reasonable and reliable.

\section{References}

[1]Zhao Hairong. Reliability Analysis and Application Efficiency Evaluation of Automatic Fire Alarm System. Northeastern University, 2009:58-70

[2] Wang Qingxi. Error analysis and research of urban tunnel fire alarm system. Wuhan University of Technology, 2010:39-49

[3] Zuo Lai. Building fire alarm system based on fuzzy genetic algorithm [J]. Computer measurement \& control, 2011,19(8): 1950-1953

[4] XING Zhixiang, CHEN Lu. Lifetime distribution and reliability research on fire detection alarming system based on MATLAB [J]. China Safety Science Journal, 2011, 21(6):48-531

[5] Guan Bin, Guan Hongmei. Efficiency Evaluation of HF Communication System Based on Fuzzy Synthetic Hierarchy Method [J]. Ship Electronic Engineering, 2014,34(2):58-62

[6] Shi Lei, Wang Xingcheng. Application of Probabilistic Neural Network in Engine Fault Diagnosis [J]. PROCESS AUTOM ATION INSTRUMENTATION,2011,32(3):33-35

[7] YUAN Jinsha, SHANG Haikun. Pattern recognition based on principal component analysis and probabilistic neural networks for partial discharge of power transformer $[\mathrm{J}]$. Electric Power Automation Equipmen,2013,33(6):27-31 